\title{
A satisfação profissional de cirurgiões-dentistas da atenção básica em um município no sul do Brasil com diretrizes municipais de saúde bucal
}

\section{The professional satisfaction of primary health care dentists in a city in southern Brazil with municipal oral health guidelines}

\author{
Júlia Guedes Alves* \\ Vitor Henrique Digmayer Romero* \\ Peterson Oliveira Boeira** \\ Tania Izabel Bighetti $i^{* *+*}$ \\ Eduardo Dickie de Castilhos ${ }^{* * * *+}$
}

\section{Resumo}

Objetivo: identificar a satisfação profissional de cirurgiões-dentistas atuantes na atenção básica do Sistema Único de Saúde em um município do sul do Brasil, que foi pioneiro na implantação de diretrizes municipais de saúde bucal. Sujeitos e método: para a coleta dos dados, foram utilizados componentes descritivos a partir de dados secundários obtidos por meio de um questionário aplicado para todos os cirurgiões-dentistas atuantes no referido serviço. Resultados: no total, 46 profissionais foram incluídos na amostra, sendo que $82,5 \%$ estavam satisfeitos em seu trabalho e, desses, o reconhecimento dos usuários do serviço foi o fator que os cirurgiões-dentistas mais associaram à felicidade. Já os fatores mais relacionados à infelicidade no trabalho são as críticas ao salário e os usuários não colaboradores; $97,8 \%$ acreditavam que seu trabalho fazia diferença na comunidade em que estavam inseridos. Conclusão: este estudo sugere que estabelecer diretrizes no processo de trabalho pode ter um impacto positivo na prática e na satisfação dos profissionais, e essas estratégias podem ser utilizadas como amparo para planejamento em gestão de saúde bucal de forma sistematizada.

Palavras-chave: Administração em saúde pública. Satisfação no emprego. Saúde pública.

\section{Introdução}

O comportamento dos profissionais vem sendo amplamente estudado, pois se sabe que a interação com o ambiente e os aspectos emocionais estão diretamente relacionados com a atuação e a qualidade do trabalho ${ }^{1}$. Os profissionais da área da saúde são um grupo que se destaca nesses estudos, tendo em vista que atuam em ambientes de alto estresse. Tais estudos se justificam pela natureza do serviço prestado pelos profissionais da saúde, de modo que a efetividade de seu trabalho tem impacto direto na saúde e na qualidade de vida do usuário do serviço ${ }^{2}$.

Dentre esses profissionais, o cirurgião-dentista é um dos que podem sofrer diversas influências do ambiente, tanto em nível físico, devido a condutas de ergonomia e biossegurança, como no nível psicológico, quanto a fatores que estão diretamente relacionados com sua satisfação profissional ${ }^{3,4}$.

A satisfação profissional está relacionada ao "estado emocional positivo resultante do prazer que se tem com as experiências do trabalho" ". Isso significa que vivências prazerosas no ambiente laboral resultam na satisfação do profissional ${ }^{5}$. Sendo assim, a reorientação no modelo de atenção - por

Cirurgiã-dentista graduada na Universidade Federal de Pelotas, Pelotas, Rio Grande do Sul, Brasil.

Acadêmico na Faculdade de Odontologia da Universidade Federal de Pelotas, Pelotas, Rio Grande do Sul, Brasil.

*** Doutorando em Materiais dentários no Programa de Pós-Graduação em Odontologia da Universidade Federal de Pelotas, Pelotas, Rio Grande do Sul, Brasil.

- Doutora em Saúde Pública pela Universidade de São Paulo e professora do Departamento de Odontologia Social e Preventiva da Faculdade de Odontologia da Universidade Federal de Pelotas, Pelotas, Rio Grande do Sul, Brasil.

****** Doutor em Epidemiologia pela Universidade Federal de Pelotas e professor do Departamento de Odontologia Social e Preventiva da Faculdade de Odontologia da Universidade Federal de Pelotas, Pelotas, Rio Grande do Sul, Brasil. 
intermédio das Diretrizes Municipais de Saúde Bucal - foi desenvolvida para assumir o compromisso de qualificação da atenção básica, conforme a Política Nacional de Saúde Bucal, promovendo, assim, no município, uma reestruturação do modelo de prática, visando a impactos positivos na saúde bucal da população, bem como no desenvolvimento do trabalho e na satisfação profissional dos cirurgiões-dentistas envolvidos ${ }^{6}$.

Todavia, a literatura ressalta que não é o profissional satisfeito e feliz que produz um bom trabalho, mas, sim, um profissional com bom rendimento e produtivo que se torna satisfeito e feliz ${ }^{7}$. Isso contextualiza a ideia de que reconhecimentos e promoções estão relacionados com satisfação e produtividade, assim como uma melhor organização da demanda e do serviço ${ }^{7}$.

Desse modo, o objetivo do presente estudo foi identificar a satisfação profissional de cirurgiões-dentistas atuantes na atenção básica do Sistema Único de Saúde (SUS) em um município do sul do Brasil, que é pioneiro na implantação de diretrizes municipais de saúde bucal.

\section{Sujeitos e método}

Este é um estudo transversal com componentes descritivos a partir de dados secundários obtidos por meio de um questionário, previamente estruturado, aplicado por um único entrevistador para todos os cirurgiões-dentistas atuantes na atenção básica do SUS de um município do sul do Brasil, que estabelece, no processo de trabalho, diretrizes municipais de saúde bucal.

$\mathrm{Na}$ seleção da amostra do estudo, foram eleitos os seguintes critérios de inclusão: ser cirurgião-dentista, regularmente inscrito no Conselho Regional de Odontologia, e estar atuando em uma Unidade Básica de Saúde (UBS) no município em questão. Foram excluídos do estudo profissionais com contrato de trabalho temporário ou em regime de cargo de confiança e que não estavam trabalhando na rede de atenção básica (como profissionais alocados em Centro de Especialidades Odontológicas) ou que não estavam em atividade no período de coleta de dados, devido a férias ou afastamentos, como licenças médica, maternidade, prêmio ou por interesse particular.

Este estudo foi previamente aprovado pelo Comitê de Ética em Pesquisa da Faculdade de Odontologia da Universidade Federal de Pelotas, sob o parecer $\mathrm{n}^{\mathrm{0}} 1.517 .439$, respeitando os princípios de bioética em pesquisa e a Resolução no 466 do Conselho Nacional de Saúde.

\section{Resulltados}

Foram entrevistados 46 cirurgiões-dentistas, distribuídos em 51 UBS. Em média, os profissionais atuavam na mesma UBS há cerca de 72,8 meses (aproximadamente 6 anos).

Quando questionados se acreditavam que seu trabalho faz diferença na comunidade em que se inserem, 97,8\% afirmaram que sim. Todavia, quando questionados se haviam conseguido reduzir as doenças bucais na comunidade, $74 \%$ responderam positivamente; enquanto $13 \%$ afirmaram que não conseguem e $13 \%$ não souberam responder.

Quando perguntado aos profissionais se eram felizes em seu trabalho, $82,5 \%$ responderam que sim, $8,7 \%$ responderam negativamente, $4,4 \%$ afirmaram ser parcialmente felizes e $4,4 \%$ não responderam ao questionamento.

Também foi perguntando aos participantes o que remetia à felicidade em seu trabalho. Dentre as respostas mais citadas, pôde-se observar que a satisfação e o reconhecimento dos usuários dos serviços foram os fatores que os cirurgiões-dentistas mais relacionaram com felicidade. Cabe salientar que o profissional poderia responder mais de uma alternativa, e quem respondeu que "não era feliz" foi contabilizado como "não se aplica" (Tabela 1).

Tabela 1 - Fatores positivos relacionados à felicidade no trabalho, citados pelos cirurgiões-dentistas da atenção básica, Pelotas, RS, 2013

\begin{tabular}{c|c}
\hline Fatores positivos do trabalho & Frequência relativa (F.A.) \\
\hline Satisfação/reconhecimento dos pacientes & $34,8 \%(16)$ \\
Promover saúde & $21,7 \%(10)$ \\
Gostar da profissão & $10,9 \%(5)$ \\
Equipe & $8,7 \%(4)$ \\
Atender determinado público & $6,5 \%(3)$ \\
Resolutividade & $6,5 \%(3)$ \\
Não respondeu & $8,7 \%(4)$ \\
Não se aplica & $6,5 \%(3)$ \\
\hline
\end{tabular}

Fonte: autores.

Quando questionados acerca do que os deixa infelizes no trabalho, críticas ao salário e usuários não colaboradores foram os fatores que apresentaram maiores frequências. Salienta-se que o profissional poderia responder mais de uma alternativa (Tabela 2). 
Tabela 2 - Fatores negativos relacionados à infelicidade no trabalho, citados pelos cirurgiões-dentistas da atenção básica, Pelotas, RS, 2013

\begin{tabular}{c|c}
\hline Fatores negativos do trabalho & $\begin{array}{c}\text { Frequência relativa } \\
\text { (F.A.) }\end{array}$ \\
\hline Salário & $15,2 \%(7)$ \\
Equipe & $10,9 \%(5)$ \\
Pacientes não colaborador & $15,2 \%(7)$ \\
Condições físicas da UBS & $6,5 \%(3)$ \\
Plano de carreira / Incentivo a atualização & $6,5 \%(3)$ \\
Não conseguir melhorar a condição de & $13,0 \%(6)$ \\
saúde da população & $2,2 \%(1)$ \\
Insegurança na UBS & $2,2 \%(1)$ \\
Desigualdade social & $2,2 \%(1)$ \\
Falta de reconhecimento & $4,3 \%(2)$ \\
Nada & $36,9 \%(17)$ \\
\hline
\end{tabular}

Fonte: autores.

Finalmente, quando indagado aos profissionais se consideravam seu trabalho rotineiro, $47,9 \%$ responderam que sim, $36,9 \%$ que não e $15,2 \%$ não responderam.

\section{Discussão}

Tendo em vista os resultados, pode-se perceber que a maioria dos cirurgiões-dentistas entrevistados apresenta-se satisfeita profissionalmente atuando no SUS ${ }^{8}$. Dado importante, uma vez que o trabalho compreende um lugar central na vida do ser humano, pois é por meio dele que se torna possível sanar as necessidades básicas, dentro de um contexto capitalista ${ }^{9}$. Desse modo, a influência que o trabalho exerce na satisfação individual de cada profissional implica fortemente em diversos âmbitos da vida. Ademais, faz-se fundamental ressaltar que a atuação do profissional, focada na assistência, de forma isolada e sem adequar-se ao contexto da população, implica fortemente nesse processo.

O processo de trabalho do odontólogo pode acarretar diversas consequências funcionais, pela ação da postura, pela vibração da caneta de alta rotação e do micromotor ${ }^{10,11}$. Desse modo, devem ser levados em consideração os domínios físicos e psicológicos dos cirurgiões-dentistas, quando avaliado seu processo de trabalho.

Um estudo ${ }^{12}$ realizado na Malásia demonstrou que houve associação entre satisfação no trabalho e inteligência emocional, e essas descobertas têm implicações para o desenvolvimento de intervenções para promover a retenção de dentistas no setor público, bem como para implementação de programas que atuem na saúde do trabalhador da área de saúde.

Em um estudo ${ }^{9}$ realizado em um município da grande Florianópolis, SC, com 16 profissionais da atenção básica (incluindo unidades tradicionais e com Estratégia de Saúde da Família), foram encon- trados diversos fatores que se assemelham aos encontrados neste trabalho. Reservando as limitações entre as metodologias, pode-se observar que a questão salarial é sempre motivo de crítica. O estudo sugere que esse fator está fortemente relacionado à formação acadêmica, caracterizando a odontologia como uma profissão de caráter liberal. Além disso, o estudo também salienta que várias falas abordaram o processo de trabalho como rotineiro e metódico, conforme também demostrado no presente estudo. Outro fator que se destaca no sofrimento profissional é a impotência diante da carência da população, evidenciando as desigualdades sociais existentes em nosso país ${ }^{9}$.

Outro trabalho ${ }^{5}$ foi realizado com 60 cirurgiões-dentistas na cidade de Bauru, SP, divididos em dois grupos: recém-formados e profissionais com algum tempo de atuação no mercado. Foi observado que os primeiros são os que se encontram mais insatisfeitos com a profissão, principalmente nas questões de honorários e realização profissional ${ }^{5}$. Sendo assim, é possível refletir que o tempo de atuação tem grande influência nas percepções individuais. Isso se deve ao contexto de formação, sendo que os profissionais mais jovens são reflexo das revoluções tecnológicas, de uma sociedade moderna em que tudo é dinâmico e ágil, com uma demanda de explanar o sucesso em público jamais vista. Desse modo, são nítidas as diferenças de percepção quanto à satisfação dos profissionais entre distintas gerações. No entanto, na presente metodologia, não foi considerado o tempo de formação do profissional, sendo uma limitação do estudo.

Cabe observar também que outros estudos ${ }^{13,14}$ destacam que as diferentes especialidades apresentam distintos graus de satisfação. Em uma pesquisa $^{13}$ no Canadá, com cerca de 330 ortodontistas, $80 \%$ estavam satisfeitos com a profissão, e um fator positivo destacado entre os principais motivos foi o relacionamento com o paciente. Já, em um estudo ${ }^{14}$ na Inglaterra, com 42 profissionais de três especialidades diferentes (cirurgia bucomaxilofacial, dentística restauradora e ortodontia), os cirurgiões bucomaxilofaciais foram os que apresentaram maiores níveis de satisfação profissional, quando comparados com os demais. Desse modo, podemos refletir que a escolha da especialidade, o ambiente de trabalho e a relação com o paciente têm significativa importância para a satisfação profissional.

Em um estudo transversal, desenvolvido em duas partes ${ }^{15,16}$, foram coletados dados acerca da força de trabalho de países desenvolvidos e subdesenvolvidos membros da World Dental Federation. Foi observado que as condições de trabalho, a educação continuada, as diretrizes que orientam o serviço e as preferências pessoais estão entre os principais fatores que afetam a prática odontológica ${ }^{16}$, resultados semelhantes aos encontrados no presente trabalho. Ainda, estudos sugerem que o aumento da conscientização e da participação em associações 
referentes à categoria é de extrema importância na luta por benefícios e superação de desafios da prática profissional ${ }^{15}$. Logo, isso pode compor um meio para ampliar a satisfação do cirurgião-dentista.

Além disso, é importante destacar que o Brasil é detentor do maior número de dentistas do mun$\mathrm{do}^{17}$, o que afeta diretamente a remuneração recebida, quando observada a competição mercadológica entre oferta e procura de profissionais. Portanto, essa problemática tem forte influência nas críticas salariais dos cirurgiões-dentistas no contexto do sistema público, principalmente quando comparado com outros países, podendo afetar a satisfação profissional ${ }^{18}$. Portanto, a gestão pública deve fornecer subsídios que estimulem o trabalhador ${ }^{19}$. Nesse sentido, o incentivo para atualizações profissionais e melhor planejamento de planos de carreira pode ser uma estratégia.

É relevante lembrar da importância do SUS no contexto social brasileiro, que assegura a assistência, a promoção e a prevenção odontológica de forma universal ${ }^{20}$. Todavia, é um sistema exclusivo do país ${ }^{20}$, de modo que estudos referentes aos impactos na satisfação dos trabalhadores do setor de odontologia são pouco fomentados, principalmente ante as divergências metodológicas entre os âmbitos de atuação público e privado, consistindo em uma limitação do trabalho. Nessa perspectiva, novas pesquisas com esse enfoque são amplamente recomendadas, a fim de subsidiar o planejamento público em saúde bucal.

\section{Conclusão}

Por meio deste estudo, percebeu-se que a maior parte dos cirurgiões-dentistas atuantes na atenção básica do SUS em um município no sul do Brasil encontra-se feliz e satisfeita com o seu trabalho. Além disso, o estudo sugere que estabelecer diretrizes no processo de trabalho pode ter um impacto positivo na prática e na satisfação profissional. Todavia, algumas questões limitantes devem ser amplamente discutidas com a categoria, principalmente no que se refere a honorários, estímulo ao reconhecimento profissional e método de trabalho em equipe. Sendo assim, essas questões podem ser utilizadas como amparo para planejamento em gestão de saúde bucal.

\section{Abstract}

Objective: to identify the professional satisfaction of dentists working in the primary health care of the public health system, in a city in southern Brazil, which was a pioneer for implementing municipal oral health guidelines. Subjects and method: the data were collected by descriptive components from secondary data obtained through a questionnaire applied to all dentists working in the referred service. Results: a total of 46 professionals were included in the sample, whereas $82.5 \%$ were satisfied with their work, and the acknowledgment from service users was the factor that these professionals mostly associated with happiness. On the other hand, the factors most associated with unhappiness in the workplace are complaints of low wages and uncooperative patients. In addition, $97.8 \%$ of the professionals believed that their work made a difference in the community in which they were inserted. Conclusion: this study suggests that establishing guidelines in the work process may have a positive impact on the practice and the professional satisfaction and that these strategies may be used as a planning support for the systemic management of oral health.

Keywords: Public health management. Job satisfaction. Public health.

\section{Referências}

1. Marqueze EC, Castro Moreno CRD. Satisfação no trabalho - uma breve revisão. Rev Bras Saúde Ocup 2005; 30(112): 69-79.

2. Gomes AR, Cruz J, Cabanelas S. Estresse ocupacional em profissionais de saúde: um estudo com enfermeiros portugueses. Psic Teor e Pesq 2009; 25(3):307-18.

3. Nunes MF, Freire MCM. Qualidade de vida de cirurgiões-dentistas que atuam em um serviço público. Rev Saúde Pública $2006 ; 40(6): 1019-26$.

4. Bonafé F, Trotta O, Campos J, Maroco J. Síndrome de burnout em dentistas do serviço público. Psychol Community Health 2012; 1(2):56-67.

5. Nicolielo J, Bastos JRM. Satisfação profissional do cirurgião-dentista conforme tempo de formado. Rev Fac Odontol Bauru 2002; 10(2):69-74.

6. Pelotas. Diretrizes da Saúde Bucal de Pelotas. 2013. [acesso em: 29 abr. 2018. Disponível em URL: http://www.pelotas. rs.gov.br/saude/arquivos/DiretrizesSaude-Bucal-de-Pelotas-2013.pdf.

7. Robbins SP. Comportamento organizacional. 9. ed. São Paulo: Prentice Hall; 2002.

8. Costa ACO, Garbin CAS, Soares GB, Gonçalves PRV, Moimaz SAS. Satisfação e o emprego no Sistema Único de Saúde na área de saúde bucal. Rev Fac Odontol Lins 2012; 22(2):13-9.

9. Gomes D, Gonçalves ASR, Pereira LS, Tavares RS, Pires DEP, Ramos FRS. Satisfação e sofrimento no trabalho do cirurgião-dentista. RFO UPF 2010; 15(2):104-10.

10. Medeiros UV, Souza MIC, Bastos LF. Odontologia do trabalho: riscos ocupacionais do cirurgião-dentista. RBO 2003; 60(4):277-80.

11. Adas Saliba N, Moimaz SAS, Prado RL, Rovida TAS, Garbin CAS. Saúde do trabalhador na odontologia: o cirurgião-dentista em foco. Pesq Bras Odontopediatria Clín Integrada 2013; 13(2):147-54.

12. Pau A, Sabri BA. Relationship between emotional intelligence and job satisfaction in newly qualified Malaysian dentists. Asia Pacific J Public Health 2015; 27(2):1733-41.

13. Roth SF, Heo G, Varhagen C, Glover KE, Major PW. Job satisfaction among Canadian orthodontists. Am J of Orthodontics and Dentofacial Orthopedics 2003; 123(6):695-700.

14. Humphris G, Lilley J, Kaney S, Broomfield D. Burnout and stress-relates factors among junior staff of three dental hospital specialities. Br Dent J 1997; 183(1):15-21. 
15. Yamalik N, Carrasco EE, Bourgeois D. Oral health workforce planning Part 1: data available in a sample of FDI member countries. Int Dent J 2013; 63(6):298-305.

16. Yamalik N, Ensaldo $\square$ Carrasco E, Cavalle E, Kell K. Oral health workforce planning part 2: figures, determinants and trends in a sample of World Dental Federation member countries. Int Dent J 2014; 64(3):117-26.

17. Saliba NA, Moimaz SAS, Garbin CAS, Diniz DG. Dentistry in Brazil: its history and current trends. J Dental Educ 2009; 73(2):225-31.

18. Cartes-Velasquez RA. Exponential growth of dental schools in Chile: effects on academic, economic and workforce issues. Braz Oral Res 2013; 27(6):471-7.

19. De Lima Trindade L, Pires De Pires DE. Implicações dos modelos assistenciais da atenção básica nas cargas de trabalho dos profissionais de saúde. Texto \& Contexto Enfermagem 2013; 22 (1):36-42.

20. Brasil. Ministério da Saúde. Diretrizes da Política Nacional de Saúde Bucal. Brasília: Ministério da Saúde; 2004.

Endereço para correspondência:

Júlia Guedes Alves

Faculdade de Odontologia, Departamento de

Odontologia Social e Preventiva

Universidade Federal de Pelotas

Rua Gonçalves Chaves, 457, Centro

96090-560 - Pelotas, RS, Brasil

E-mail: juliaguedesa@outlook.com

Recebido: 15/05/18. Aceito: 04/07/18. 